\title{
Searches for Beyond Standard Model Higgs Bosons with ATLAS*
}

\author{
Nikolaos Rompotis on behalf of the ATLAS Collaboration \\ University of Washington, \\ Physics Department, Box 351560, Seattle WA 98195-1560
}

\begin{abstract}
The search for evidence of beyond Standard Model Higgs bosons is an integral part of the Higgs boson studies at the LHC. This article reviews recent beyond Standard Model Higgs boson searches using $2-5 \mathrm{fb}^{-1}$ of $7 \mathrm{TeV}$ LHC proton-proton collision data recorded by the ATLAS detector. No significant deviations from the background expectations are found and corresponding constraints on physics beyond the Standard Model are obtained.
\end{abstract}

PACS numbers: PACS numbers come here

\section{Introduction}

The recent observation of a new particle with mass $125-126 \mathrm{GeV}$ at the LHC $[1,2]$ opens a new era for particle physics. The detailed study of the production and decay modes of the new particle provides invaluable input to answer the question of whether this is indeed the long-sought Standard Model (SM) Higgs boson [3-7]. The first measurements indicate that the new particle is indeed compatible with the SM Higgs boson [8], nevertheless, many more measurements and data will be needed to extract reliable conclusions. This task is further complicated by the fact that many beyond SM physics scenarios include a SM-like Higgs boson, which is part of an extended scalar sector. In that case, searches for beyond SM Higgs bosons are very interesting, since they provide direct information on a possibly extended scalar sector, and hence they are complementary to the precise measurements of the properties of the new particle.

This article reviews some of the recent searches for beyond SM Higgs bosons in proton-proton collision data at $7 \mathrm{TeV}$ centre-of-mass energy produced by the LHC [9] and recorded by the ATLAS detector [10].

\footnotetext{
* Presented at the 42nd International Symposium on Multiparticle Dynamics, Kielce, Poland, September 2012.
} 


\section{Higgs Bosons in beyond SM physics scenarios}

The scalar sector of the SM is composed by a complex Higgs doublet, which after the electroweak symmetry breaking (EWKSB) leaves a single scalar boson in the theory. Possible extensions of the scalar sector are restricted, due to the fact that in general they violate the "custodial symmetry" of the $\mathrm{SM}^{1}$. The addition of doublets or singlets naturally conserves the custodial symmetry and hence it comprises an attractive idea on which beyond SM scenarios can be constructed.

The introduction of one additional Higgs doublet defines a class of models, which are collectively known as 2 Higgs doublet models (2HDM) [12]. The type II 2HDM, a 2HDM in which the first doublet couples to up-type fermions and the second doublet couples to down-type fermions, has been extensively studied. One of the reasons, is that the Minimal Supersymmetric Standard Model (MSSM) $[13,14]$ is a specific case of a type II 2HDM. Supersymmetric theories, like the MSSM, have been introduced to explain a number of problems of the SM (e.g. see [15]) and they enjoy high popularity among the high-energy physics community. In a 2HDM, there are five Higgs bosons after the EWKSB. More specifically, there is one CP-odd boson, $A$, two CP-even bosons $2, h$ and $H$, and two charged Higgs bosons $H^{ \pm}$. The number of free parameters of the theory in the specific case of the MSSM are just two at tree level. These parameters are usually chosen to be $m_{A}$ and $\tan \beta$ or $m_{H^{ \pm}}$and $\tan \beta$, where $\tan \beta \equiv u_{1} / u_{2}$ and $u_{1}\left(u_{2}\right)$ is the vacuum expectation value of the Higgs doublet coupling to up- (down-) type fermions. It is worth noting that the MSSM is compatible with the assumption that there is a SM-like Higgs boson with mass $125-126 \mathrm{GeV}[16,17]$. In ATLAS, searches for $A / H / h \rightarrow \tau \tau / \mu \mu$ [18], $H^{ \pm} \rightarrow \tau^{ \pm} \nu$ [19] and $H^{ \pm} \rightarrow c s$ [20] are inspired by the MSSM (and 2HDM in general) and the first two of these will be discussed in the next section.

More complicated choices for the scalar sector have also been studied and searches in ATLAS data are available for a variety of different signatures: scalar sector with light CP-odd particles [21,22], doubly charged Higgs boson [23], "fermiophobic" Higgs bosons [24], Higgs boson in a model with a fourth generation of fermions [25], and Higgs bosons decaying to long-lived particles $[26,27]$. In the following, only two of these more exotic cases will be discussed in more detail $[22,26]$.

\footnotetext{
1 The name "custodial symmetry" will be used to denote the experimental fact that the quantity $\rho \equiv m_{W}^{2} /\left(m_{Z}^{2} \cos ^{2} \theta\right) \simeq 1$, where $m_{W(Z)}$ is the $W(Z)$ boson mass and $\theta$ the weak mixing angle, see also [11].

2 The notation is such that $m_{h}<m_{H}$.
} 


\section{MSSM-inspired searches}

Over large part of the parameter space of a general $2 \mathrm{HDM}$, and hence also the MSSM, the decays of the Higgs bosons to third generation fermions are enhanced $[12,15]$. The neutral Higgs boson decays of $A / H / h \rightarrow \tau \tau$ are experimentally atractive, due to the progress in $\tau$ decays reconstruction and the fact that the branching fraction to $\tau \tau$ is about $10 \%$ for the parameter space that is relevant for this search. In a recent search using $4.7-4.8 \mathrm{fb}^{-1}$ of data [18], neutral Higgs bosons decaying to a $\tau \tau$ pair are searched for, including cases where: both $\tau$ s decay hadronically; one $\tau$ decays to an electron or muon plus neutrinos and the other hadronically; and finally one $\tau$ decays to an electron plus neutrinos and the other $\tau$ to a muon plus neutrinos. In addition to the more sensitive $\tau \tau$ modes, the decay to a di-muon pair has been considered. This decay mode suffers from a very low branching fraction, which is about $10^{-4}$, but profits from high mass resolution and high rejection of reducible backgrounds. No excess is observed with respect to the expectation from SM background processes. The results of the search can be interpreted in the $m_{h}^{\max }$ scenario of the MSSM [28] on the $m_{A^{-}} \tan \beta$ space as shown in Figure 1(a).

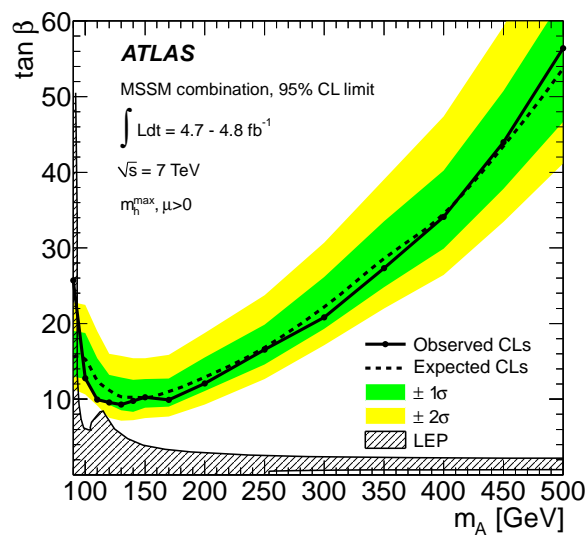

(a) $A / H / h \rightarrow \tau \tau / \mu \mu$ search

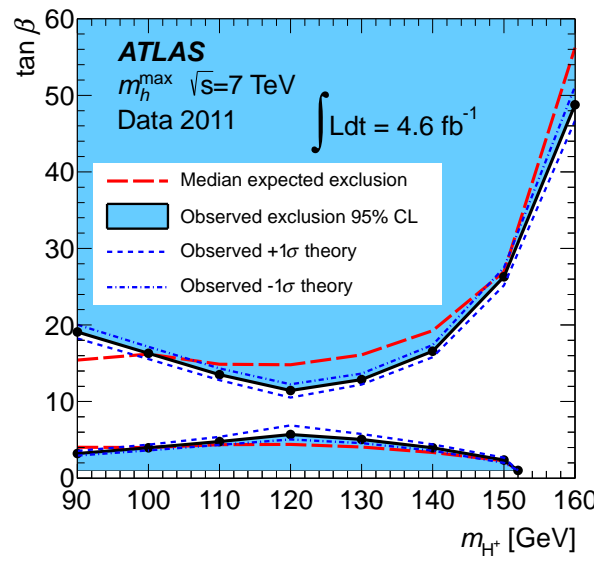

(b) $H^{ \pm} \rightarrow \tau \nu$ search

Fig. 1. Interpretation of the $A / H / h \rightarrow \tau \tau / \mu \mu$ search [18] (a) and the $H^{ \pm} \rightarrow \tau \nu$ search [19] (b) in the $m_{h}^{\max }$ scenario of the MSSM [28]. The 95\% confidence level exclusion limits for the neutral and charged Higgs bosons are shown in the $m_{A^{-}}$ $\tan \beta$ and the $m_{H^{ \pm}}-\tan \beta$ planes.

Searches for a light charged Higgs boson, produced in top quark decays $\left(t \rightarrow b H^{ \pm}\right)$and decaying as $H^{ \pm} \rightarrow \tau^{ \pm} \nu$, with $4.6 \mathrm{fb}^{-1}$ of ATLAS data are described in Reference [19]. The final state $t \bar{t} \rightarrow b \bar{b} W^{\mp} H^{ \pm}$is characterised 
by the decay products of the $W$ and the $\tau$ from the charged Higgs decay. More specifically, the following cases have been examined: " $\tau+$ jets": $\tau$ decaying hadronically and $W \rightarrow$ jets; " $\tau+$ lepton": $\tau$ decaying hadronically and $W \rightarrow e \nu / \mu \nu$; "lepton+jets": $\tau \rightarrow e+$ neutrinos $/ \mu+$ neutrinos and $W \rightarrow$ jets. The combination of all these three channels shows good agreement with the expectation from the SM processes. The results of the search can be interpreted $^{3}$ in the $m_{h}^{\max }$ scenario of the MSSM [28] on the $m_{H^{ \pm}}-\tan \beta$ space as shown in Figure 1(b).

\section{Exotic signature searches}

A number of beyond SM physics scenarios include light CP-odd scalar particles in which the Higgs boson can decay to, e.g. see [29]. A search for a Higgs boson decaying to a pair of light $(\sim 100 \mathrm{MeV})$, CP-odd particles, $a$, with each of them decaying to a di-photon pair: $H \rightarrow a a \rightarrow(\gamma \gamma)+(\gamma \gamma)$ with $4.9 \mathrm{fb}^{-1}$ of data is described in Reference [22]. The small mass of $a$ particles with respect to the Higgs boson mass leads to highly boosted di-photon pairs, which are reconstructed as a single photon in the electromangetic calorimeter. The analysis follows the SM $H \rightarrow \gamma \gamma$ search [30], but with a modified photon identification to allow for the broader showers of the boosted di-photon pairs with respect to the shower of a single photon. No excess is observed with respect to the SM background processes and exclusion limits are extracted for the cross section of Higgs boson production times the branching fraction $\mathrm{BR}(H \rightarrow a a \rightarrow 4 \gamma)$ under various assumptions for the $a$ particle mass, see Figure 2 and Reference [22].

In "hidden valley" models [31] there is a hidden sector, which communicates with the rest of the SM particles through a communicator particle. Assuming that the Higgs boson plays the role of the communicator particle it can decay to hidden sector particles. The search described in Reference [26] looks for Higgs boson decays to long-lived hidden valley pions, $H \rightarrow \pi_{V} \pi_{V}$, in $1.9 \mathrm{fb}^{-1}$ of data. These particles travel several metres inside the detector and they subsequently decay as $\pi_{V} \rightarrow b \bar{b} / c \bar{c} / \tau \tau$. Their decay products are detected in the muon system using dedicated reconstruction algorithms. Triggering on such kind of events is highly non-trivial, since the usual reconstruction algorithms are designed to reject events with objects that do not originate from the interaction point. For this reason a dedicated trigger was developped, using muon system clusters only. No deviation is observed with respect to the SM background processes and exclusion limits

\footnotetext{
${ }^{3}$ Both neutral and charged MSSM Higgs searches can have other, more general interpretations than the $m_{h}^{\max }$ scenario of the MSSM. For more details see References $[18,19]$.
} 


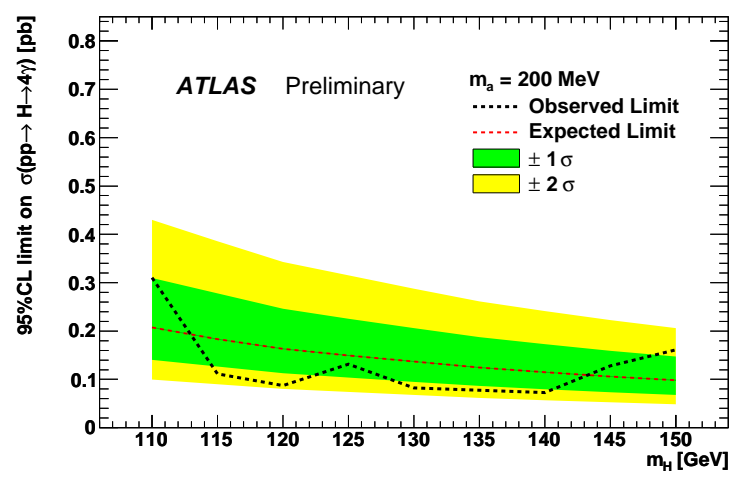

Fig. 2. Exclusion limits at the $95 \%$ confidence level (CL) for the Higgs boson cross section times $\mathrm{BR}(H \rightarrow a a \rightarrow 4 \gamma)$ assuming that the mass of the CP-odd particle $a$ is $200 \mathrm{MeV}[22]$.

for the Higgs boson production cross section are derived, which are shown in Figure 3 .

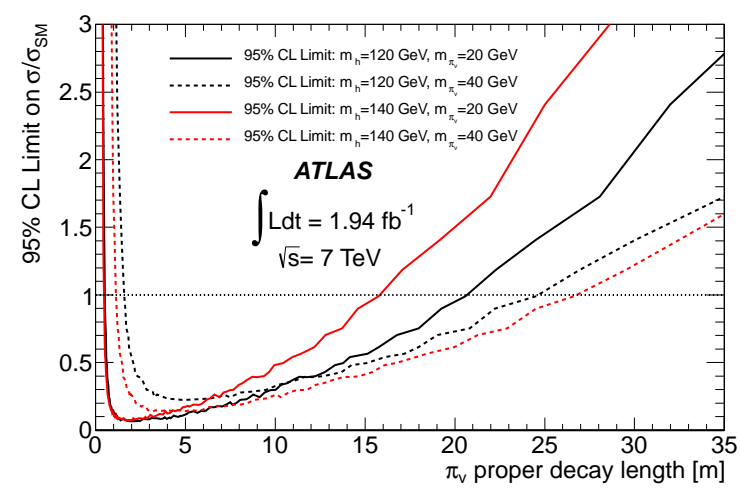

Fig. 3. Exclusion limits on the cross section of Higgs boson production divided by its value in the SM, assuming that the Higgs boson decays exclusively to $\pi_{V} \pi_{V}$, for various choices of Higgs boson and $\pi_{V}$ masses [26].

\section{Concluding remarks}

ATLAS searches for beyond SM Higgs bosons increase the discovery potential of beyond SM physics at the LHC. Moreover, they contribute towards the effort to understand whether the recently observed $125-126 \mathrm{GeV}$ particle is indeed the SM Higgs boson. 


\section{REFERENCES}

[1] ATLAS Collaboration. Phys. Lett., B 716:1, 2012.

[2] CMS Collaboration. Phys. Lett., B 716:30, 2012.

[3] P. W. Higgs. Phys. Lett., 12:132, 1964.

[4] P. W. Higgs. Phys. Rev. Lett., 13:508, 1964.

[5] P. W. Higgs. Phys. Rev., 145:1156, 1966.

[6] F. Englert and R. Brout. Phys. Rev. Lett., 13:321, 1964.

[7] G. Guralnik, C. Hagen and T. Kibble. Phys. Rev. Lett., 13:585, 1964.

[8] ATLAS Collaboration. ATLAS-CONF-2012-127, http://cdsweb.cern.ch/record/1476765.

[9] L. Evans and P. Bryant. JINST, 3:S08001, 2008.

[10] ATLAS Collaboration. JINST, 3:S08003, 2008.

[11] J. Gunion, H. Haber, G. Kane, and S. Dawson. Front.Phys., 80:1-448, 1990.

[12] G. Branco et al. Phys.Rept., 516:1-102, 2012.

[13] H. P. Nilles. Phys. Rep., 110:1, 1984.

[14] H. E. Haber and G. L. Kane. Phys. Rep., 117:75, 1985.

[15] A. Djouadi. Phys. Rep., 459:1, 2008.

[16] S. Heinemeyer, O. Stal and G. Weiglein. Phys. Lett., B 710:201, 2012.

[17] A. Arbey, M. Battaglia, A. Djouadi and F. Mahmoudi. JHEP, 1209:107, 2012.

[18] ATLAS Collaboration. Submitted to JHEP, arXiv:1211.6956, 2012.

[19] ATLAS Collaboration. JHEP, 1206:039, 2012.

[20] ATLAS Collaboration. ATLAS-CONF-2011-094, https://cdsweb.cern.ch/record/1367737.

[21] ATLAS Collaboration. ATLAS-CONF-2011-020, https://cdsweb.cern.ch/record/1336749.

[22] ATLAS Collaboration. ATLAS-CONF-2012-127, https://cdsweb.cern.ch/record/1460391.

[23] ATLAS Collaboration. Phys. Rev. D, 85:032004, Feb 2012.

[24] ATLAS Collaboration. Eur.Phys.J., C72:2157, 2012.

[25] ATLAS Collaboration. ATLAS-CONF-2011-135, https://cdsweb.cern.ch/record/1383838.

[26] ATLAS Collaboration. Phys.Rev.Lett., 108:251801, 2012.

[27] ATLAS Collaboration. Submitted to Physics Letters B, arXiv:1210.0435, 2012.

[28] M. Carena, S. Heinemeyer, C. Wagner and G. Weiglein. Eur. Phys. J., C 26:601, 2003.

[29] B. Dobrescu, G. Landsberg, and K. Matchev. Phys.Rev., D63:075003, 2001.

[30] ATLAS Collaboration. Phys.Rev.Lett., 108:111803, 2012.

[31] M. J. Strassler and K. M. Zurek. Phys.Lett., B651:374-379, 2007. 